\title{
Influence of microstructures on mechanical behaviours of SiC nanowires: a molecular dynamics study
}

\author{
Jun Wang ${ }^{1,2,3,4}$, Chunsheng Lu ${ }^{1}$, Qi Wang ${ }^{3}$, Pan Xiao ${ }^{4}$, Fujiu Ke ${ }^{2,4}$, \\ Yilong Bai ${ }^{4}$, Yaogen Shen ${ }^{5}$, Xiaozhou Liao ${ }^{6}$ and Huajian $\mathrm{Gao}^{7}$ \\ ${ }^{1}$ Department of Mechanical Engineering, Curtin University, Perth, WA 6845, Australia \\ ${ }^{2}$ School of Physics and Nuclear Energy Engineering, Beihang University, Beijing 100191, \\ People's Republic of China \\ ${ }^{3}$ School of Aeronautics Science and Engineering, Beihang University, Beijing 100191, \\ People's Republic of China \\ ${ }^{4}$ State Key Laboratory of Nonlinear Mechanics (LNM), Institute of Mechanics, \\ Chinese Academy of Sciences, Beijing 100190, People's Republic of China \\ ${ }^{5}$ Department of Mechanical and Biomedical Engineering, City University of Hong Kong, \\ Kowloon, Hong Kong, People's Republic of China \\ ${ }^{6}$ School of Aerospace, Mechanical and Mechatronic Engineering, University of Sydney, \\ Sydney, NSW 2006, Australia \\ ${ }^{7}$ School of Engineering, Brown University, Providence, RI 02912, USA \\ E-mail: C.Lu@curtin.edu.au and bhwangqi@sina.com
}

Received 2 August 2011, in final form 24 October 2011

Published 14 December 2011

Online at stacks.iop.org/Nano/23/025703

\begin{abstract}
The tensile behaviours of [111]-oriented $\mathrm{SiC}$ nanowires with various microstructures are investigated by using molecular dynamics simulations. The results revealed the influence of microstructures on the brittleness and plasticity of $\mathrm{SiC}$ nanowires. Plastic deformation is mainly induced by the anti-parallel sliding of $3 \mathrm{C}$ grains along an intergranular amorphous film parallel to the (11) plane and inclined at an angle of $19.47^{\circ}$ with respect to the nanowire axis. Our study suggests that the wide dispersion of mechanical properties of $\mathrm{SiC}$ nanowires observed in experiments might be attributed to their diverse microstructures.
\end{abstract}

(Some figures may appear in colour only in the online journal)

\section{Introduction}

Over the past few years, $\mathrm{SiC}$ has attracted increasing interests due to its potential applications in various electronic and structural devices [1]. The lack of ductility of $\mathrm{SiC}$ at room temperature, however, is a serious concern. It is known that the formation of nanostructures is a promising strategy to improve the capacity of deformation. Recently, significant progress has been achieved in developing new nanostructured $\mathrm{SiC}$ nanowires (NWs). For example, a brittle to ductile transition with large strain plasticity was detected in $\mathrm{SiC}$ NWs with local strain reaching nearly $2 \%$, which is in distinct contrast to typical strain levels of $0.1-0.2 \%$ or even smaller in their bulk counterparts [2, 3]. Furthermore, superplastic deformation with local strain exceeding $200 \%$ has been observed under uniaxial tensile loading of SiC NWs [4]. Based on the existing theory, the observed super-plasticity might be due to dislocation nucleation, propagation and amorphization developed in cubic (3C) single-crystal segments [4]. It is worth noting, however, that only elastic deformation and brittle failure were revealed in most analyses on tensile and bending deformation in $\mathrm{SiC}$ [5-8]. In a recent study, it is shown that the brittle to ductile transition could be triggered by an amorphous shell which covers a crystal core at temperatures beyond $700 \mathrm{~K}$ [9]. However, the mechanism of large plastic deformation at room temperature remains unclear. On the other hand, the experimentally reported values of Young's modulus of [111]-oriented $\mathrm{SiC} \mathrm{NWs}$ vary widely in the range from 20 to $750 \mathrm{GPa}$ [10-13], without a consensus value even for a specific, fixed wire dimension. A similar 
(a)

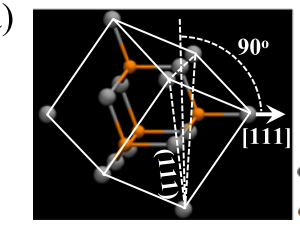

(c)
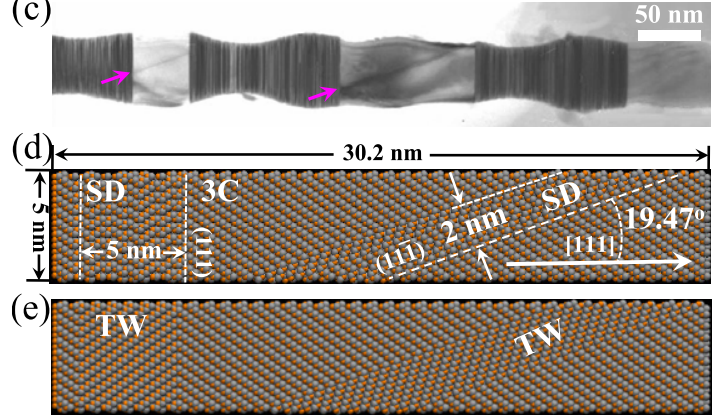

(f)

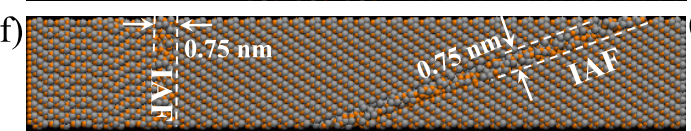

(b)

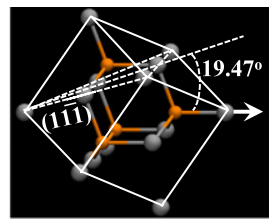

(j)

(j) As:

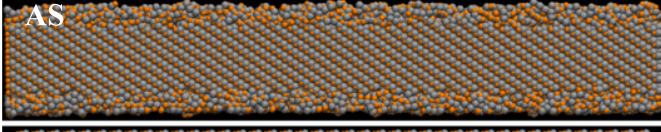

(k)

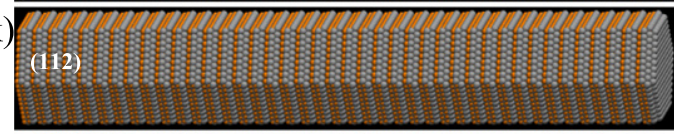

(1)

(m)

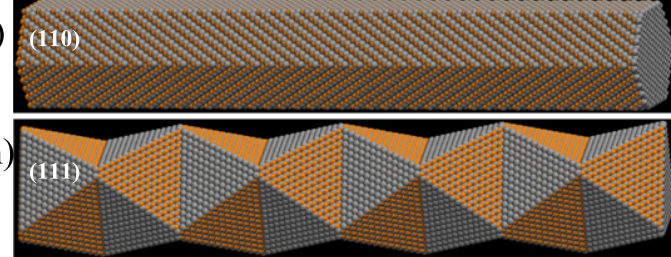

Figure 1. Illustrations of possible microstructures in SiC NWs. (a) and (b) show $90^{\circ}$ stacking and $19.47^{\circ}$ stacking planes, respectively. (c) is an SiC NW adopted from [4], where darker strips and lighter regions correspond to defects occurring at $90^{\circ}$ and $3 \mathrm{C}$ segments, respectively. Thin black slashes in 3C segments indicate defects with an inclination angle of $19.47^{\circ}$ (indicated by arrows). (d)-(f) are schematic diagrams of stacking defects (SD), twins (TW) and intergranular amorphous films (IAF) at inclination angles of $90^{\circ}$ and $19.47^{\circ}$ to the axis. (g) shows interfaces where point defects are generated randomly. (h) and (i) illustrate vacancy cases with $5 \%$ surface density at $90^{\circ}$ and $19.47^{\circ}$, respectively. (j)-(m) show patterns of NWs containing amorphous shell (AS), (112) facets, (110) facets and Wulff twinning blocks.

phenomenon also exists in their strengths. In addition to differences in experimental protocols and testing error, so far there is no satisfactory understanding on the wide dispersion of mechanical properties observed in experiments.

It is generally believed that mechanical properties of a material critically depend on its internal structures at different length scales. We note that the microstructures of $\mathrm{SiC} \mathrm{NWs}$ synthesized in laboratories usually consist of [111]-oriented 3C-structured segments [14], stacking defects (SD) [4] and twins (TW) [15]. Moreover, an intergranular amorphous film (IAF) with a thickness of $\sim 0.6-0.9 \mathrm{~nm}$ between grains was formed as a result of aluminium added as a sintering aid [16]. Since aluminium is usually used to restrict their lateral growth, IAFs most likely exist in SiC NWs [17]. For [111]-oriented 3C-structured segments, the following side morphologies have been reported: an ultra-thin amorphous shell [14, 18], (112) or (110) facets [6,19] and Wulff twinning blocks with (111) facets [20]. Si and $\mathrm{C}$ vacancies were also recognized as intrinsic point defects in $\mathrm{SiC}$ nanostructures [21, 22]. The influence of a specific microstructure on mechanical properties can hardly be evaluated by experiments since an individual $\mathrm{SiC}$ NW may contain one or more such microstructures. Such a gap can be at least partially addressed with numerical simulations.

In a recent paper, we have shown that IAFs can result in large plastic deformation of $\mathrm{SiC} \mathrm{NWs}$ at room temperature [23]. The present paper is aimed to use molecular dynamics simulations to further investigate the influence of various microstructures on mechanical behaviours of SiC NWs, focusing on (i) the thermal stability of plastic deformation associated with IAFs and (ii) the wide dispersion of mechanical properties that result from microstructural anisotropy. This paper is organized as follows. In section 2, $\mathrm{SiC}$ NWs with various microstructures are constructed and the adopted simulation techniques are introduced. Section 3 is then dedicated to a detailed discussion of mechanical behaviours and properties of $\mathrm{SiC}$ NWs with different microstructures. Factors that may have caused the large plastic deformation as well as the wide dispersion of mechanical properties are discussed in section 4. Finally, some concluding remarks are given in section 5 .

\section{Simulation procedures}

\subsection{Microstructures}

In a cubic crystal, there are four close-packed planes: (11) (111), (111) and (111). The former three planes form an angle of $19.47^{\circ}$ with respect to the [111] orientation, while the latter is perpendicular to the [111] orientation. Considering that stacking faults occur usually on close-packed planes like (111) or the equivalent in $3 \mathrm{C}-\mathrm{SiC}$, and the axis of an individual $\mathrm{NW}$ is along the [111] orientation, there are only two relative types of spatial configurations: stacking faults occurring on (111) and (111) form angles of $19.47^{\circ}$ and $90^{\circ}$ with respect to the axis of an NW, respectively; see figures 1(a) and (b). This can be clearly seen in the transmission electronic microscopy image shown in figure 1(c) [4]. SD, TW and IAF can occur at either angle, as illustrated in figures $1(\mathrm{~d})-(\mathrm{f})$. For the convenience of discussion hereafter, we define IAF- $90^{\circ}$ as IAFs at an angle of $90^{\circ}$ to the axis. Similar abbreviations are also used in other microstructures.

To investigate the influence of SD, TW and IAF on mechanical properties of $\mathrm{SiC} \mathrm{NWs}$, a diverse array of 
Table 1. Parameters used in the interaction potential for SiC NWs, as shown in equations (1)-(6), where $\lambda=5.0 \AA$, $\xi=3.0 \AA$ and $r_{\mathrm{c}}=7.35 \AA$ [25]. The effective charge on $\mathrm{Si}$ and $\mathrm{C}$ are $1.201 e$ and $-1.201 e$, respectively ( $e$ being the unit of electronic charge).

\begin{tabular}{lllllll}
\hline & & $\eta_{i j}$ & $H_{i j}\left(\mathrm{eV} \AA^{\eta}\right)$ & $D_{i j}\left(e^{2} \AA^{3}\right)$ & $W_{i j}\left(\mathrm{eV} \AA^{6}\right)$ & \\
\hline Two-body & $\mathrm{Si}-\mathrm{Si}$ & 7 & 23.67291 & 2.1636 & 0 & \\
& $\mathrm{Si}-\mathrm{C}$ & 9 & 447.09026 & 1.0818 & 61.4694 & \\
& $\mathrm{C}-\mathrm{C}$ & 7 & 471.74538 & 0 & 0 & \\
\hline \multirow{5}{*}{ Three-body } & & $B_{i j k}(\mathrm{eV})$ & $\bar{\theta}_{j i k}(\mathrm{deg})$ & $C_{j i k}$ & $\gamma(\AA)$ & $r_{0}(\AA)$ \\
& $\mathrm{Si}-\mathrm{C}-\mathrm{Si}$ & 9.003 & 109.47 & 5.0 & 1.0 & 2.9 \\
& $\mathrm{C}-\mathrm{Si}-\mathrm{C}$ & 9.003 & 109.47 & 5.0 & 1.0 & 2.9 \\
\hline
\end{tabular}

microstructures were considered in this study. For SD-19.47 and TW-19.47 $7^{\circ}$, four different thicknesses of SD/TW $(2,3,4$ and $5 \mathrm{~nm}$ ) were chosen, while in the cases of SD-90 and TW$90^{\circ}$, the selected thicknesses of SD/TW were 5,10 and $15 \mathrm{~nm}$, corresponding to compositions of $1 / 6,1 / 3$ and $1 / 2$, respectively. To study the mechanical behaviour induced by an IAF, we consider an IAF with thickness equal to $0.75 \mathrm{~nm}$, which lies in the middle of the measured ranges [16]. The 3C-crystal segments were generated by an ordered sequence of three basic structural modules of tetrahedral bonding in $\mathrm{SiC}[24]$, SDs by randomly ordered sequences of these modules and TWs by anti-ordered sequences in comparison with that of $3 \mathrm{C}$ segments. The IAF was simulated by filling an amorphous film into a corresponding carved region in a prepared NW. To examine the thermal stability of IAF, its thickness was set to be $2 \mathrm{~nm}$ while the temperature varied from 300 to $2400 \mathrm{~K}$. Point defects were modelled by randomly removing atoms from interfaces (dashed lines in figure $1(\mathrm{~g})$ ) between 3C segments and SDs. Figures $1(\mathrm{~h})$ and (i) show cases of 5\% vacancies at $90^{\circ}$ and $19.47^{\circ}$, respectively. Samples have a round cross section with a diameter of $5 \mathrm{~nm}$.

Moreover, five surface morphologies have been investigated: round lateral section, amorphous shell (AS), (112) facets, (110) facets and Wulff twinning blocks with (111) facets. The latter four morphologies are shown in figures 1(j)(m). Each of these has a cross-sectional dimension of about $5 \mathrm{~nm}$. Thicknesses of the amorphous shell and $3 \mathrm{C}$ core are chosen to be 1 and $1.5 \mathrm{~nm}$, respectively. The aspect ratio of $\mathrm{SiC}$ NWs is about 6:1. To ensure traction-free boundary conditions, a vacuum region of $3 \mathrm{~nm}$ was attached on the outside of the lateral surface. Periodic boundary conditions were applied in all directions.

\subsection{Molecular dynamics simulation}

To describe interactions among atoms, an empirical potential is used which consists of two-body ( $\mathrm{Si}-\mathrm{Si}, \mathrm{Si}-\mathrm{C}$ and $\mathrm{C}-\mathrm{C})$ and three-body ( $\mathrm{Si}-\mathrm{C}-\mathrm{Si}$ and $\mathrm{C}-\mathrm{Si}-\mathrm{C})$ covalent interactions in the form

$$
V=\sum_{i<j} V_{i j}^{(2)}\left(r_{i j}\right)+\sum_{i, j<k} V_{j i k}^{(3)}\left(r_{i j}, r_{i k}\right)
$$

where $r_{i j}$ is the distance between atoms $i$ and $j$ [25]. The twobody part can be written as

$$
V_{i j}^{(2)}\left(r_{i j}\right)=\frac{H_{i j}}{r_{i j}^{\eta_{i j}}}+\frac{Z_{i} Z_{j}}{r_{i j}} \mathrm{e}^{-r_{i j} / \lambda}-\frac{D_{i j}}{2 r_{i j}^{4}} \mathrm{e}^{-r_{i j} / \xi}-\frac{W_{i j}}{r_{i j}^{6}},
$$

where $H_{i j}$ is the strength of steric repulsion, $Z_{i}$ is the charge, $D_{i j}$ is the strength of charge-dipole attraction, $W_{i j}$ is the van der Waals interaction strength, $\eta_{i j}$ is the exponent of steric repulsion term, and $\lambda$ and $\xi$ are the screening lengths of Coulomb and charge-dipole terms, respectively.

The three-body term in equation (1) combines both spatial and angular dependences in the form

$$
V_{j i k}^{(3)}\left(r_{i j}, r_{i k}\right)=R^{(3)}\left(r_{i j}, r_{i k}\right) P^{(3)}\left(\theta_{j i k}\right),
$$

where

$$
\begin{aligned}
& R^{(3)}\left(r_{i j}, r_{i k}\right)=B_{j i k} \exp \left(\frac{\gamma}{r_{i j}-r_{0}}+\frac{\gamma}{r_{i k}-r_{0}}\right) \\
& \quad \times \Theta\left(r_{0}-r_{i j}\right) \Theta\left(r_{0}-r_{i k}\right)
\end{aligned}
$$

and

$$
P^{(3)}\left(\theta_{j i k}\right)=\frac{\left(\cos \theta_{j i k}-\cos \bar{\theta}_{j i k}\right)^{2}}{1+C_{j i k}\left(\cos \theta_{j i k}-\cos \bar{\theta}_{j i k}\right)^{2}} .
$$

In these expressions, $B_{j i k}$ is the strength of interaction, $\theta_{j i k}$ is the angle between $r_{i j}$ and $r_{i k}, C_{j i k}$ and $\bar{\theta}_{j i k}$ are constants and $\Theta\left(r_{0}-r_{i j}\right)$ indicates a step function. The two-body interaction is truncated at $r_{\mathrm{c}}(7.35 \AA)$. To keep the potential and its first derivative continuous at $r_{\mathrm{c}}$, the two-body interaction is shifted with

$$
\begin{aligned}
& V_{(i j)}^{(2 \text { shifted })}(r) \\
& =\left\{\begin{array}{c}
V_{i j}^{(2)}(r)-V_{i j}^{(2)}\left(r_{\mathrm{c}}\right)-\left(r-r_{\mathrm{c}}\right)\left(\mathrm{d} V_{i j}^{(2)}(r) / \mathrm{d} r\right)_{r=r_{\mathrm{c}}} \\
r \leqslant r_{\mathrm{c}} \\
0 \quad r>r_{\mathrm{c}} .
\end{array}\right.
\end{aligned}
$$

The parameter values of these two-body and three-body potentials are listed in table 1 [25].

The motion of atoms is integrated using a Verlet leapfrog algorithm to yield velocities and positions with a time step of 2 fs. To obtain free-standing configurations, samples without amorphous composition are relaxed for $20 \mathrm{ps}$ before stretching. Those containing amorphous composition are relaxed in a time range of $0.5-2.0 \mathrm{~ns}$ at desired temperatures to ensure statistical steady states have been reached.

A quasi-static loading scheme is applied to simulate and obtain tensile deformation and mechanical properties of SiC NWs. The strain increment along [111] is achieved in two steps. First, a modified isothermal-isobaric ensemble is used to stretch NWs with a strain rate of $0.001 \mathrm{ps}^{-1}$ for $1 \mathrm{ps}$, which results in a nominal strain of $0.1 \%$ at each deformation increment [26]. The axial strain is then held and NWs are relaxed for 6 ps via a canonical ensemble [27]. 


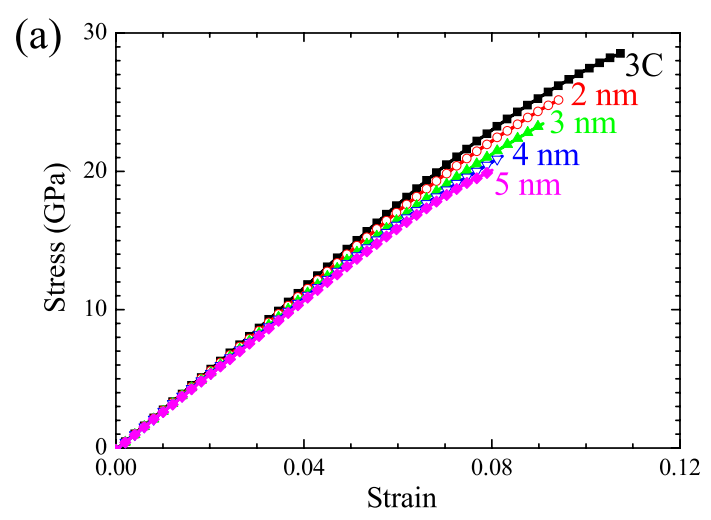

(c)

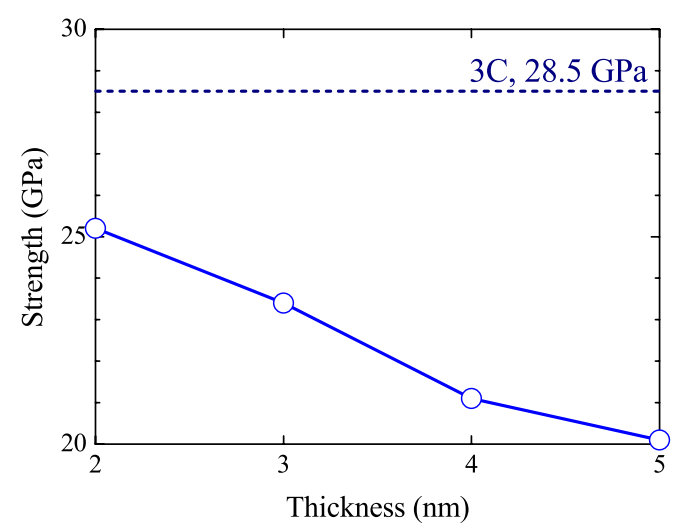

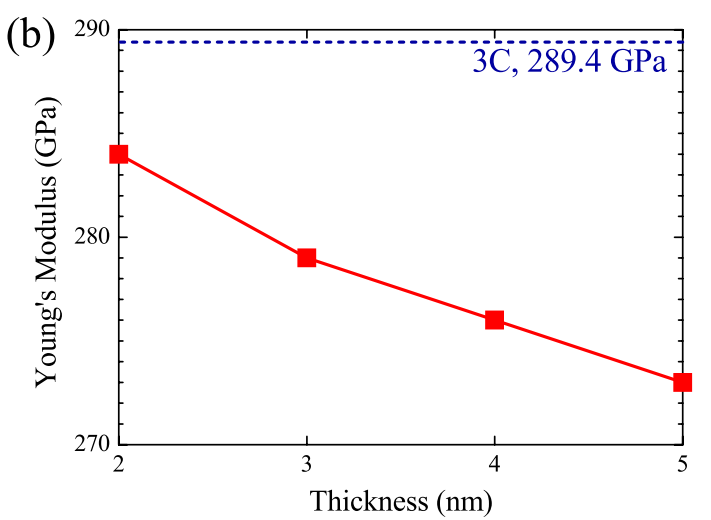

(d)

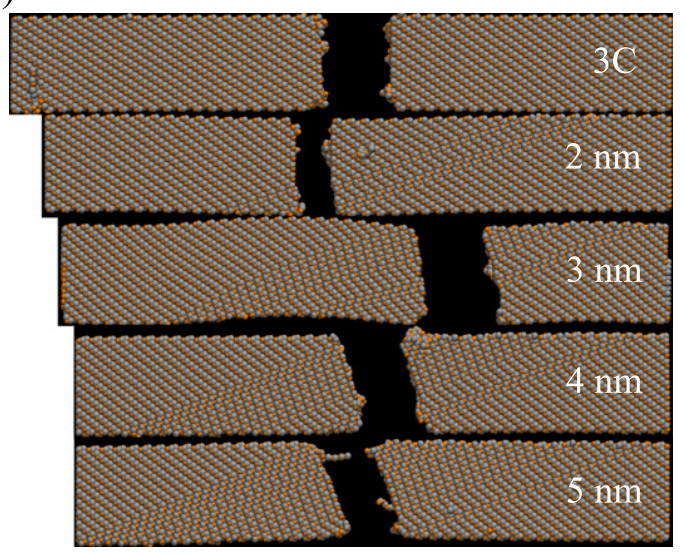

Figure 2. Mechanical behaviour of SiC NWs with stacking defects at $19.47^{\circ}$. (a) shows stress-strain curves of SiC NWs with thicknesses of stacking defects from 2 to $5 \mathrm{~nm}$. (b) and (c) show Young's modulus and tensile strength versus thickness of stacking defects. Patterns after fracture are shown in (d).

The stress tensor is calculated by a modified virial formula which is independent of the kinetic energy or mass transfer and can be identified with the Cauchy stress [28]. The Young's modulus is fitted by using the prior $2 \%$ stage of a stressstrain curve. More details of numerical techniques were discussed in [29]. All calculations were carried out with the DL_POLY2.20 package [27].

\subsection{Validation of the interatomic potential}

Taking the [111]-oriented single-crystal 3C NWs with lateral dimension of $5 \mathrm{~nm}$ as a reference, the Young's modulus predicted by the interatomic potential is $289.4 \mathrm{GPa}$, which falls in the middle of experimental results between 20 and $750 \mathrm{GPa}$ [10-13]. In contrast, the Tersoff potential seems to predict a larger value of the Young's modulus of $370 \mathrm{GPa}$ with a similar lateral dimension [30]. The present interatomic potential can be used to model structural, elastic and dynamical properties of bulk 3C-SiC, amorphous as well as the liquid states of $\mathrm{SiC}$. The predicted melting point of bulk $3 \mathrm{C}-\mathrm{SiC}$ is $3250 \pm 50 \mathrm{~K}$, which agrees well with the experimental result of $3103 \pm 40 \mathrm{~K}$. The energetics of several polymorphs (3C, wurtzite and rock salt) and the $3 \mathrm{C}$ to rock salt transformation pressure obtained by the potential are also in agreement with experimental observations and $a b$ initio simulations [25]. The current potential also successfully characterizes the influence of amorphous grain boundaries on mechanical behaviours [31]. These facts are essential to the prediction of mechanical properties related to the amorphous composition in $\mathrm{SiC}$ NWs and its corresponding thermal stability.

\section{Simulation results}

\subsection{Stacking defects}

Stress-strain curves of SD-19.47 with SD thickness from 2 to $5 \mathrm{~nm}$ are shown in figure 2(a). To illustrate its influence on mechanical properties, the tensile test on a single-crystal 3C NW is taken as reference. All samples show an elastic response before brittle fracture. The single-crystal 3C NW has the highest Young's modulus, tensile strength and elongation of $289.4 \mathrm{GPa}, 28.5 \mathrm{GPa}$ and $10.7 \%$, respectively. With increasing thickness of SD- $19.47^{\circ}$ from 2 to $5 \mathrm{~nm}$, the Young's modulus decreases by $3.9 \%$ from 283.9 to $272.8 \mathrm{GPa}$; see figure 2(b). Moreover, the tensile strength shows a substantial drop of $20.2 \%$ from 25.2 to $20.1 \mathrm{GPa}$, as shown in figure 2(c). Fractured patterns reveal that intragranular fracture dominates the failure behaviour of SiC NWs with SD-19.47 (figure 2(d)).

The composition of SD- $90^{\circ}$ causes a slight reduction of the Young's modulus of SiC NWs; see figure 3(a). Specifically, as the composition of SD increases from 0 to 50\%, the Young's modulus only decreases by $1.0 \%$ in contrast to $3 \mathrm{C}$ NWs. 


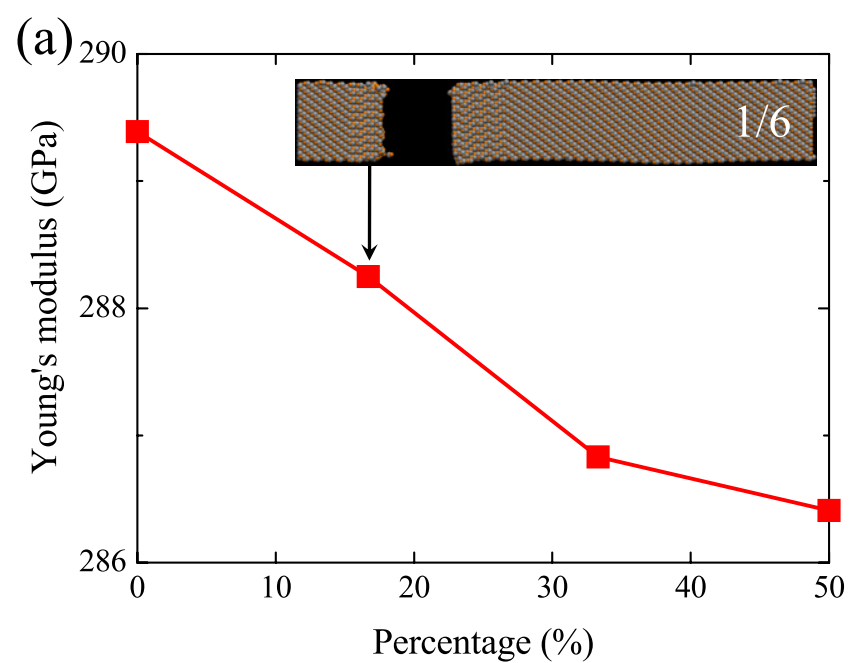

(b)

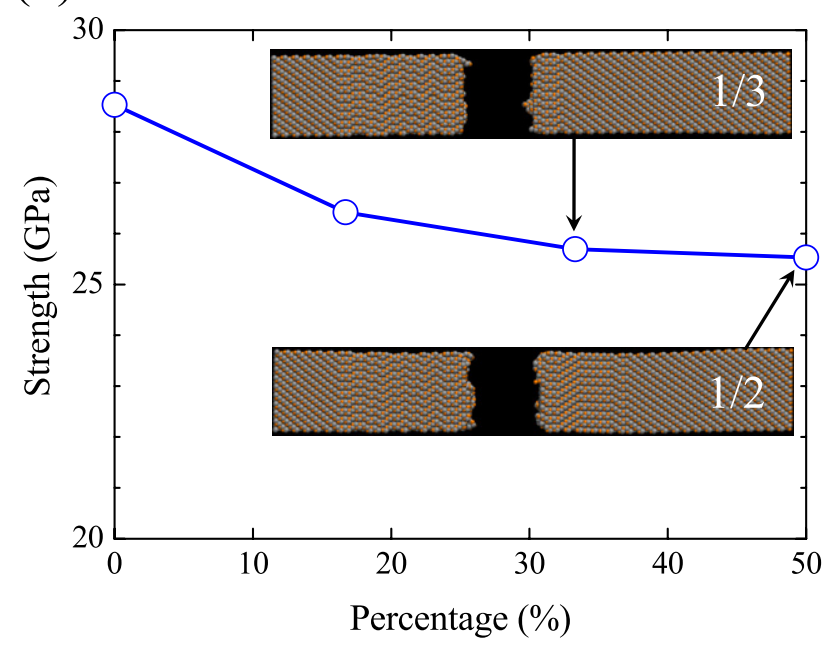

Figure 3. Mechanical properties of SiC NWs as stacking defects happen at $90^{\circ}$. (a) and (b) show Young's modulus and tensile strength versus percentage of stacking defects, respectively. Insets are patterns after fracture.

Here, it is worth noting that the $3 \mathrm{C} \mathrm{NW}$ can be regarded as a special case with $0 \%$ composition of SD- $90^{\circ}$. However, SD$90^{\circ}$ has a stronger influence on the tensile strength. As the SD composition increases to $50 \%$, the tensile strength decreases by $10.5 \%$ from 28.5 to $25.5 \mathrm{GPa}$; see figure $3(\mathrm{~b})$. The insets in figure 3 are fractured patterns of SD- $90^{\circ} \mathrm{NWs}$, which show that brittle failure occurs at an SD- $90^{\circ}$ region. It is of interest to see that only brittle failure is detected in all SD- $90^{\circ} \mathrm{NWs}$.

\subsection{Twins}

Twins have no obvious influence on the intrinsic brittle behaviour of SiC NWs whether they occur at the orientation of $19.47^{\circ}$ or $90^{\circ}$. Thus, for the convenience of comparison, a $3 \mathrm{C} \mathrm{NW}$ is taken as the reference case with $0 \%$ of twins. It is seen in figure 4(a) that, as the thickness of TW-19.47 increases to $5 \mathrm{~nm}$, the Young's modulus of NWs decreases by $5.2 \%$ from 289.4 to $274.3 \mathrm{GPa}$. The tensile strength, shown in figure $4(\mathrm{~b})$, shows a reduction of $25.3 \%$ from 28.5 to $21.0 \mathrm{GPa}$. The fractured patterns (see the insets in figure 4) indicate that
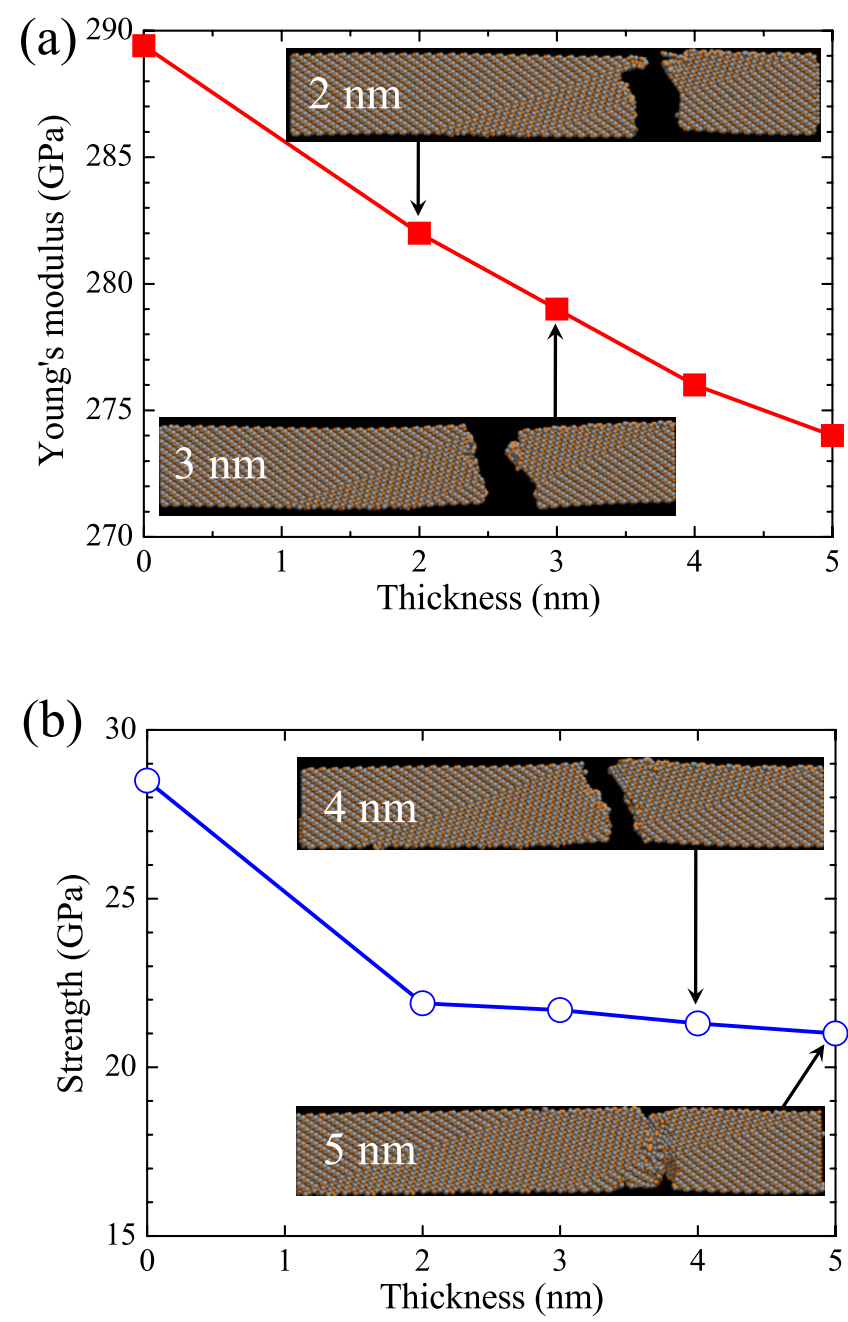

Figure 4. Mechanical properties of SiC NWs as twins occur at $19.47^{\circ}$. (a) and (b) show that Young's modulus and tensile strength vary with thickness of twins. Insets are snapshots after fracture.

intragranular fracture happens in all the NWs containing TW$19.47^{\circ}$.

TW- $90^{\circ}$ has a slight influence on the Young's modulus of $\mathrm{SiC}$ NWs. As the composition of $\mathrm{TW}-90^{\circ}$ increases to $50 \%$, the Young's modulus $(288.5 \mathrm{GPa})$ only decreases by $0.3 \%$ in contrast to that with $3 \mathrm{C}(289.4 \mathrm{GPa})$; see figure 5(a). Moreover, the tensile strength (shown in figure $5(\mathrm{~b})$ ) decreases by $6.3 \%$ from 28.5 to $26.7 \mathrm{GPa}$. The insets in figure 5 are snapshots of patterns after fracture, which show that brittle failure always happens at the twin boundaries.

\subsection{Intergranular amorphous film}

Recently, we have shown that IAF has a significant influence on the mechanical behaviour of SiC NWs [23]. The IAF$90^{\circ}$ causes brittle failure as a tensile stress reaches $12.1 \mathrm{GPa}$ with a strain of $4.5 \%$. It is unexpected that IAF- $19.47^{\circ}$ causes qualitatively different tensile behaviours: Plastic deformation occurs after the tensile strength $(10.1 \mathrm{GPa})$ is reached and the maximum elongation is up to $20.9 \%$ (figure 6(a)), which is 4.6 times the corresponding value of $4.5 \%$ in the case of 


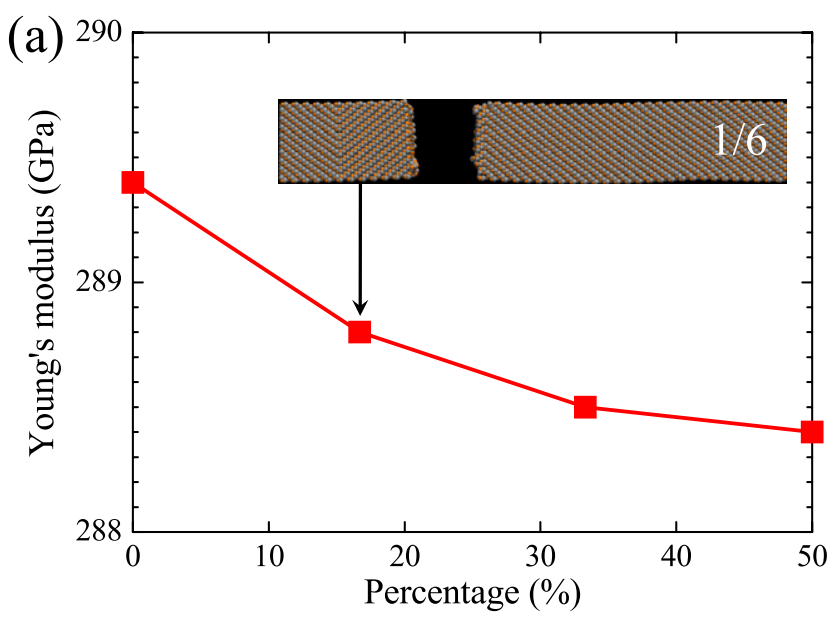

(b)

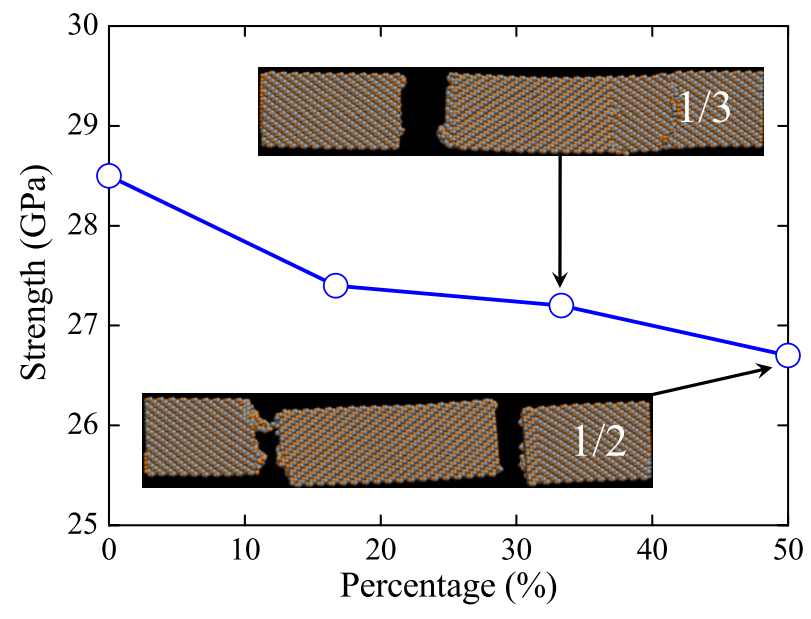

Figure 5. Mechanical properties of SiC NWs as twins happen at $90^{\circ}$. (a) and (b) indicate reduction of Young's modulus and tensile strength as composition of the twin increases. Insets are patterns after fracture.

IAF- $90^{\circ}$. The plastic deformation is achieved through the antiparallel sliding of two $3 \mathrm{C}$ grains along the (11) plane, as shown in figure 6(b). The tensile response shows sawtooth jumps that result from stretching, breaking and re-forming of $\mathrm{Si}-\mathrm{C}$ bonds in IAF; see figures 6(c)-(e). Specifically, as strain increases from $8.5 \%$ to $9.6 \%$, stretching of $\mathrm{Si}-\mathrm{C}$ bonds in IAF dominates deformation (from $\mathrm{C}$ to $\mathrm{D}$ in figure 6(a)). As they reach the tensile limit, the stretched bonds break and lead to an abrupt stress drop until a new bond forms (from D to E in figure 6(a)). Thus, the anti-parallel sliding of two 3C grains results in repeated sawtooth jumps. It is worth noting that the local unloading curve at point $\mathrm{D}$ is consistent with the corresponding loading curve at point $\mathrm{C}$. That is, the sawtooth stage of $\mathrm{C}$ to $\mathrm{D}$ originates from the elastic stretching of $\mathrm{Si}-\mathrm{C}$ bonds in IAF. Furthermore, the slope of upswing portion of each sawtooth (from $\mathrm{C}$ to D) is similar to that of the elastic regime of the stress-strain curve, indicating that mechanical properties of SiC NWs are determined by the stretching limit of $\mathrm{Si}-\mathrm{C}$ bonds in IAF. Repetition of sawtooth jumping behaviours produces a large plastic deformation until brittle failure of the right $3 \mathrm{C}$ grain; see figure 6(f).
We have also investigated the thermal stability of IAF and temperature-dependent mechanical properties of samples in a temperature range of 300-2400 K. For temperatures below $500 \mathrm{~K}, \mathrm{IAF}-19.47^{\circ}$ and IAF- $90^{\circ}$ are stable. Beyond $500 \mathrm{~K}$, crystallization occurs in IAF- $90^{\circ}$, which eventually changes to stacking defects. The critical temperature at initiation of crystallization in IAF- $19.47^{\circ}$ is $700 \mathrm{~K}$ and the corresponding patterns are shown in figure 7(a). Moreover, relaxation at a higher temperature leads to surface reconstruction. Take the case at $2400 \mathrm{~K}$ as an example; the previous round lateral shape gradually changes to a hexagonal one. Surface atoms move spontaneously to form (110) facets (see figure 7(a) and refer to figure 1(1) for comparison).

The tensile test on relaxed samples shows that $700 \mathrm{~K}$ is the critical temperature, as illustrated in figure 7(b). Below $700 \mathrm{~K}$, plastic deformation is attributed to the anti-parallel sliding of 3C grains along IAF- $19.47^{\circ}$. Above $700 \mathrm{~K}$, however, the intragranular brittle fracture dominates the failure behaviour of SiC NWs, as shown by typical patterns in the insets of figure $7(\mathrm{~b})$.

It is also seen that the Young's modulus and tensile strength of SiC NWs with IAFs are temperature-sensitive, exhibiting ascending, stable and descending stages as temperature increases. In particular, as temperature increases from 300 to $700 \mathrm{~K}$, the Young's modulus increases from 194.4 to $230.2 \mathrm{GPa}$. In the temperature range of 700 to $1300 \mathrm{~K}$, the Young's modulus is stable and ranges between 219.6 and $230.2 \mathrm{GPa}$. For temperature beyond $1300 \mathrm{~K}$, the Young's modulus decreases from 228.7 to $180.9 \mathrm{GPa}$; see figure 7(c). The tensile strength has a similar tendency, as shown in figure 7(d). In the temperature range of 300-700 K, the tensile strength increases from 6.1 to $11.9 \mathrm{GPa}$. From then on and until the temperature reaches $1600 \mathrm{~K}$, the tensile strength is stable between 9.2 and $12.4 \mathrm{GPa}$. Beyond $1600 \mathrm{~K}$, the tensile strength declines and achieves a minimum value of $6.5 \mathrm{GPa}$ at $1800 \mathrm{~K}$.

\subsection{Point defects}

The SD- $19.47^{\circ}$ can be regarded as a special case of PD- $19.47^{\circ}$ at $0 \%$ concentration. Similarly, as the concentration of PD$90^{\circ}$ reduces to $0 \%, \mathrm{PD}-90^{\circ}$ degenerates to $\mathrm{SD}-90^{\circ}$. With the concentration of PD- $19.47^{\circ}$ reaching $15 \%$, the Young's modulus decreases by $4.4 \%$ from 283.9 to $271.4 \mathrm{GPa}$, as shown in figure $8(\mathrm{a})$. The tensile strength decreases by $25.8 \%$ from 25.2 to $18.7 \mathrm{GPa}$, see figure 8(b). Moreover, intragranular fracture occurs independent of the concentration of point defects; see the insets in figure 8 . The PD- $90^{\circ}$ has a negligible influence on the Young's modulus but substantial effect on the tensile strength. With the concentration of PD- $90^{\circ}$ increasing to $15 \%$, the Young's modulus only decreases by $0.9 \%$ from 288.3 to $285.7 \mathrm{GPa}$, see figure 9 (a). However, the tensile strength reduces dramatically by $28.4 \%$ from 26.4 to $18.9 \mathrm{GPa}$ in the concentration range, as shown in figure 9(b). Fractured patterns (see the insets in figure 9) indicate that brittle failure happens at the interface between $3 \mathrm{C}$ and stacking defects. 
(a)

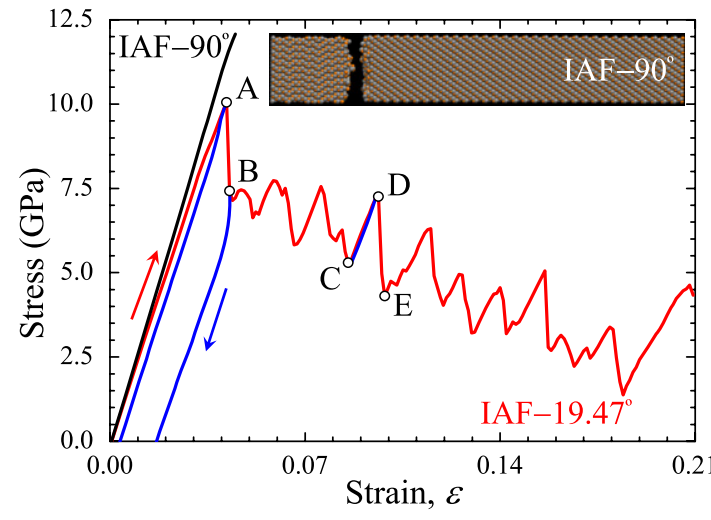

(b) $\varepsilon=8.5 \%$

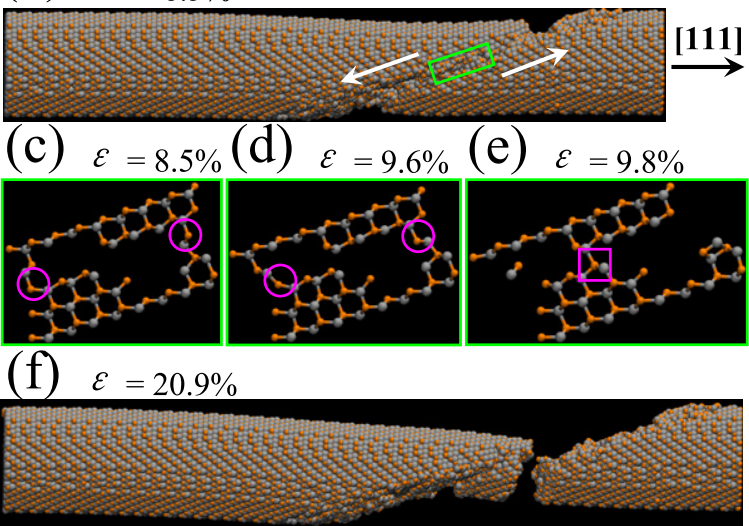

Figure 6. Large plastic deformation caused by IAF- $19.47^{\circ}$. (a) Stress-strain curve, where the inset is the pattern of IAF- $90^{\circ}$ after fracture. (b) Anti-parallel sliding of two grains along IAF, where arrows indicate sliding directions. (c)-(e) demonstrate micro-mechanisms of a sawtooth jump in the stress-strain response (points C, D and E in (a)), in which stretching and re-forming of bonds are highlighted by circles and a box, respectively. (f) A snapshot of IAF- $19.47^{\circ}$ after failure.

(a)
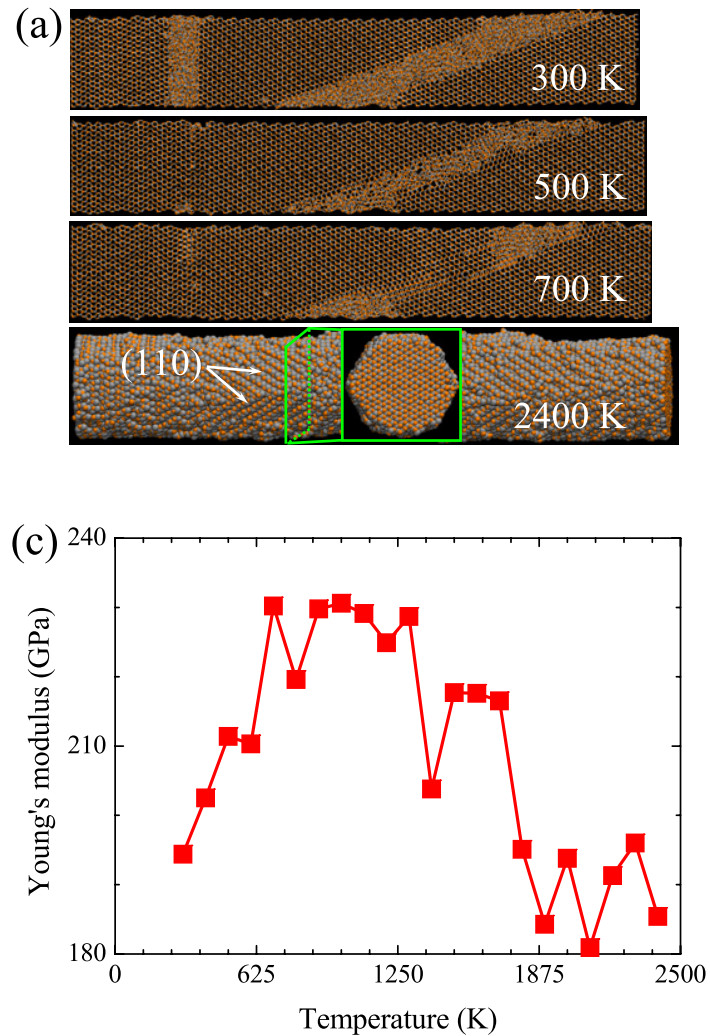

(b)

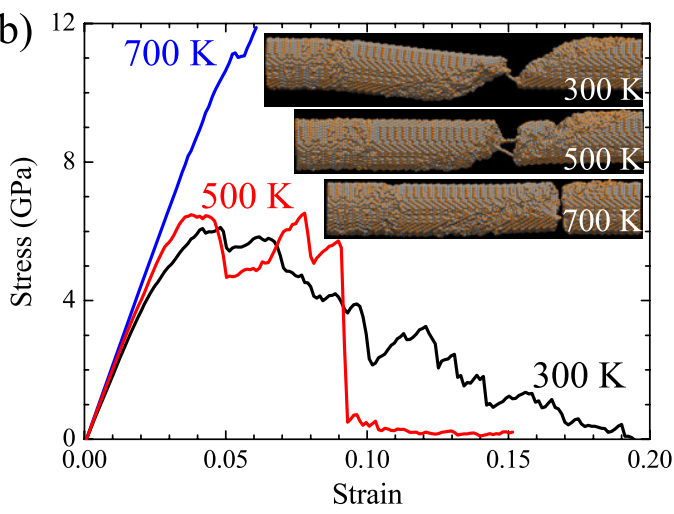

(d)

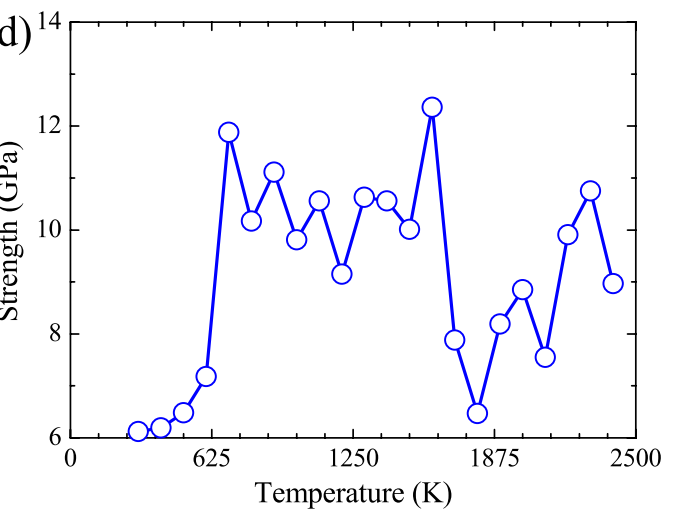

Figure 7. Thermal stability of intergranular amorphous film and corresponding temperature-dependent mechanical properties. (a) Snapshots after relaxation at various temperatures. (b) Stress-strain curves of SiC NWs containing intergranular amorphous films at 300,500 and $700 \mathrm{~K}$. Insets are patterns after fracture. (c) and (d) show variation of Young's modulus and tensile strength with temperature.

\subsection{Surface morphology}

Five surface morphologies have been analysed: three singlecrystal 3C NWs with round cross section, (110) and (112) facets, one with Wulff twinning blocks and one with an amorphous shell. As shown in figure 10, brittle failure occurs at $300 \mathrm{~K}$ in all five cases. The (110)-facetted NW generates the highest Young's modulus, tensile strength and elongation of $318.6 \mathrm{GPa}, 33.4 \mathrm{GPa}$ and $12.1 \%$. The amorphous shell brings the lowest Young's modulus and tensile strength of 158.4 and $12.4 \mathrm{GPa}$, while the Wulff blocks cause the smallest elongation of $7.9 \%$. As a result, the discrepancy of the Young's modulus can be as high as a factor of 2. Moreover, the dispersions of tensile strength and elongation are 2.7 and 1.5 times, respectively.

Fractured patterns with different surface morphologies are shown as insets in figure 10. In the case of Wulff blocks, 

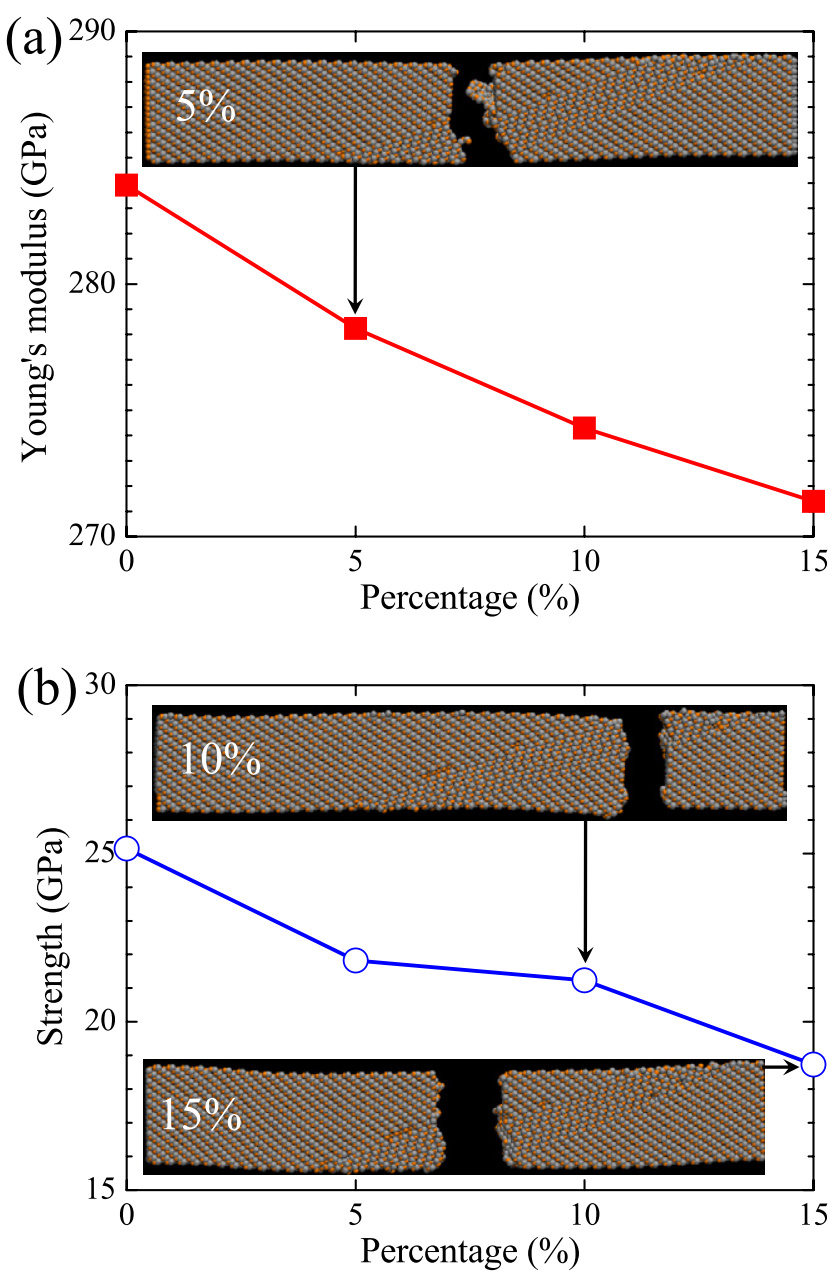

Figure 8. Mechanical properties of $\mathrm{SiC} \mathrm{NWs} \mathrm{as} \mathrm{point} \mathrm{defects} \mathrm{occur}$ at the interface between stacking defects and 3C grains $\left(19.47^{\circ}\right)$. (a) and (b) show Young's modulus and tensile strength versus the density of point defects. Insets are snapshots after fracture.

brittle failure occurs at the twin boundary of two neighbouring blocks. All the other four cases show brittle failure between two neighbouring (111) planes. Here, it is worth noting that the round NW is just the case of a single-crystal $3 \mathrm{C} \mathrm{NW}$.

\section{Discussion}

\subsection{Large plastic deformation and thermal stability}

Among all the microstructures under investigation, IAF- $19.47^{\circ}$ is the only one which induces large plastic deformation. Investigation on the thermal stability of IAF shows that crystallization occurs as temperature increases beyond $700 \mathrm{~K}$, resulting in the loss of plasticity. The sintering process in synthesizing $\mathrm{SiC}$ NWs is usually performed under a higher temperature which may trigger the crystallization of IAF. Thus, SiC NWs containing residual IAFs will show plastic deformation, while others exhibit only brittle behaviours. This explains why plastic deformation is so rarely observed in $\mathrm{SiC}$ NWs. With a reduction in amorphous composition due to crystallization, both the Young's modulus and tensile strength increase as temperature rises from 300 to $700 \mathrm{~K}$. Following
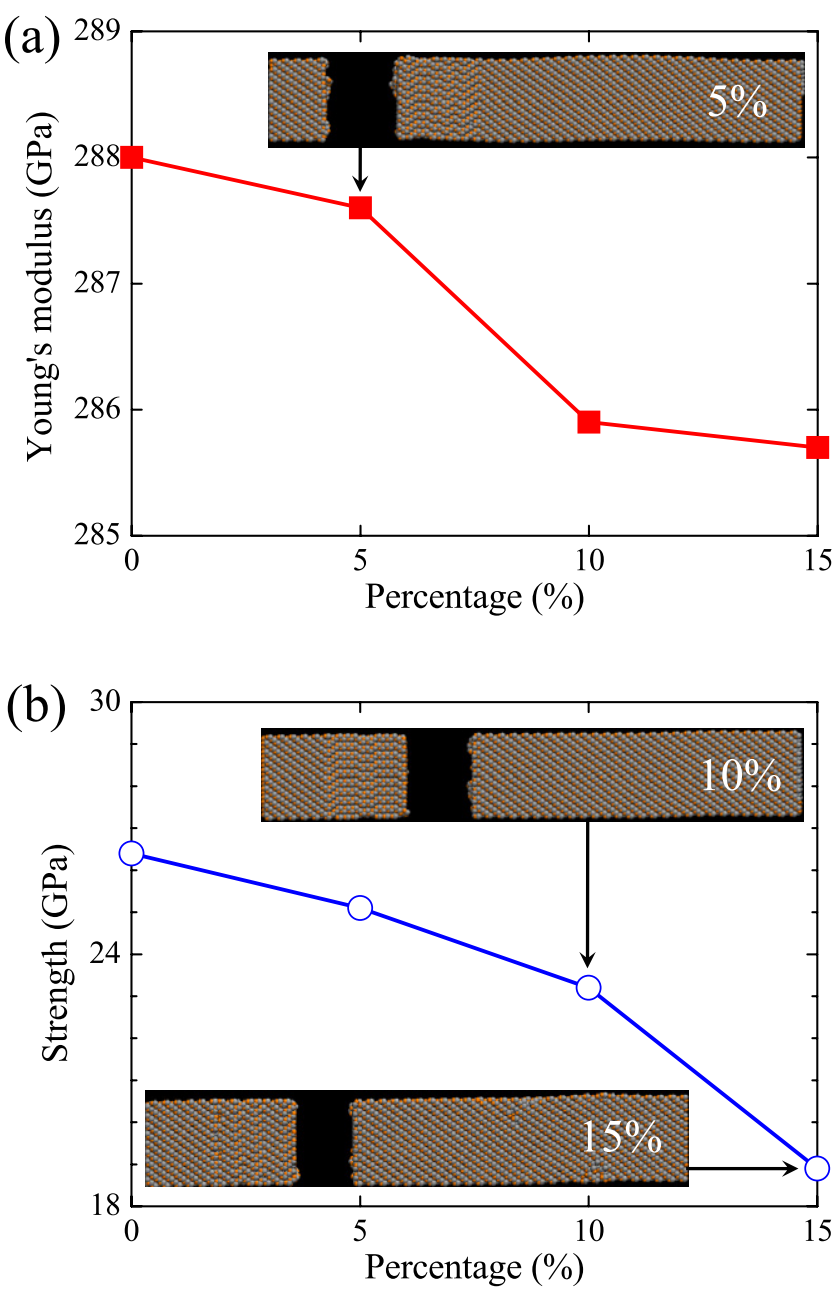

Figure 9. Mechanical properties of SiC NWs as point defects occur at the interface between stacking defects and $3 \mathrm{C}$ grains $\left(90^{\circ}\right)$. (a) and (b) show Young's modulus and tensile strength versus the density of point defects. Insets are snapshots after fracture.

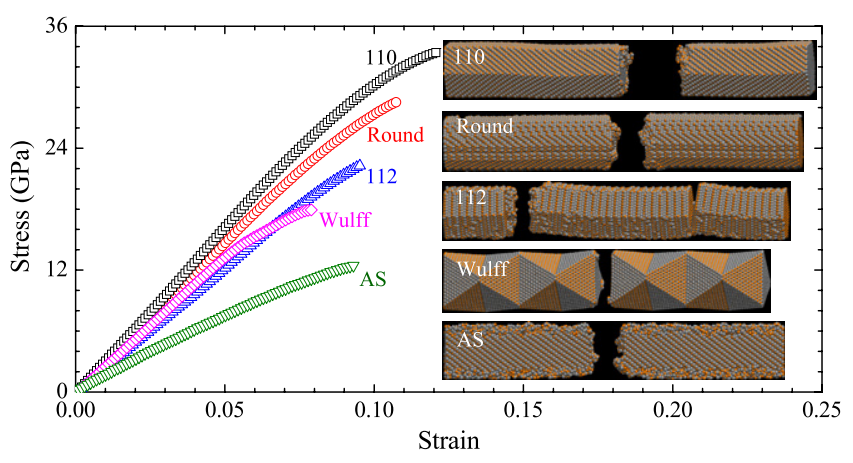

Figure 10. Stress-strain curves of SiC NWs with (110) facets, round cross section, (112) facets, Wulff twinning blocks and amorphous shell. Insets show fractured patterns.

the ascending stage, there is a stage of stable Young's modulus and tensile strength, where crystallization is saturated. As temperature rises beyond $1600 \mathrm{~K}$, thermal softening dominates the mechanical behaviour and renders weaker mechanical properties. Annealing at a higher temperature 
(e.g. $2400 \mathrm{~K}$ ) causes faster atomic diffusion and spontaneous surface reconstruction generates (110) facets which are the minimum energy state in [111]-oriented 3C NWs. This is also shown by the fact that the (110)-facetted NW possesses the highest Young's modulus and tensile strength: the more stable a state, the stiffer it is and the higher its strength. Furthermore, it is worth noting that IAFs with higher density and various spatial locations can form IAF networks, known as amorphous grain boundaries, which may also contribute to local plasticity [31]. Many metallic NWs also exhibit large plastic deformation [32-34]. However, in contrast to the plasticity of $\mathrm{SiC}$ NWs which is caused primarily by antiparallel sliding of $3 \mathrm{C}$ grains along IAFs, the deformation of metallic NWs is usually related to dislocation activities.

\subsection{Dispersion of mechanical properties in SiC NWs}

Our study reveals that the mechanical properties of SiC NWs can be extraordinarily sensitive to their microstructures. The largest Young's modulus is generated by the (110)-facetted $\mathrm{NW}$, which is twice the corresponding value for an NW with an amorphous shell. The former shows the strongest tensile strength while the weakest strength occurs in the IAF- $19.47^{\circ}$ sample with a thickness of $2 \mathrm{~nm}$, with a discrepancy in strength of about 5.5 times. Individual $\mathrm{SiC}$ NWs may contain different microstructures, which may have caused the large dispersion of mechanical properties observed in experiments. Also, these properties are also dependent on the size of a particular microstructure. Taking SD- $19.47^{\circ}$ as an example, as the SD thickness increases from 2 to $5 \mathrm{~nm}$, the Young's modulus and the tensile strength decrease by $3.9 \%$ and $20.2 \%$, respectively. The wide dispersion of mechanical properties can be attributed to microstructural anisotropy $[35,36]$. In a single crystal, physical and mechanical properties often vary with orientation. The increase of non-[111]-oriented composition in $\mathrm{SiC}$ NWs causes reductions in the Young's modulus and tensile strength. Moreover, it is seen that the Young's modulus shows a smoother attenuation in contrast to the tensile strength as the thickness of a particular defect increases. This is because the Young's modulus is only associated with the initial, linear elastic deformation of a material, on which the compositional increase of a particular microstructure has little influence. In contrast, the tensile strength is related to the failure of atomic bonds as the peak stress is reached, and various microstructures can weaken the tensile strength of $\mathrm{SiC}$ NWs to different extents. The Young's modulus of SiC NWs can also vary with the lateral dimensions of the NWs [8], which is usually attributed to the higher stress state near the surface area of NWs [30, 37].

\section{Conclusions}

Based on molecular dynamics simulations, we have shown that various possible microstructures in $\mathrm{SiC}$ NWs can have significant influences on their mechanical behaviours and properties. These microstructures can be responsible for qualitatively opposite ductile versus brittle behaviours as well as large dispersions in mechanical properties of $\mathrm{SiC}$
NWs, such as the Young's modulus, tensile strength and elongation. The main conclusions of the present study can be summarized as follows. (i) Large plastic deformation occurs as a result of anti-parallel sliding of $3 \mathrm{C}$ grains along IAF- $19.47^{\circ}$, and all other microstructures generate essentially brittle behaviours. The IAF- $19.47^{\circ}$ is thermally stable below $700 \mathrm{~K}$. (ii) Wide dispersion of mechanical properties can be attributed to microstructural anisotropy. The present study provides a better understanding of experimentally observed large plastic deformation and wide dispersion of mechanical properties in $\mathrm{SiC}$ NWs and similar nanostructured materials, as well as potential guidance on microstructural design of ceramics with tailored deforming properties.

\section{Acknowledgments}

This work has been supported by the Australian Research Council (grant no DP0985450), the National Natural Science Foundation of China (grant nos 11172024, 10732090, 10932011, 11072014, 10972218 and 11021262), the Fundamental Research Funds for the Central Universities (YWF11-03-Q-505 and YWF-11-03-Q-573), the National Basic Research Program of China (2007CB814803), the China Postdoctoral Science Foundation (2011M500211) and the US National Science Foundation (CMMI-0758535). Computations were performed on computer clusters at the CNIC Supercomputing Centre, iVEC through the use of advanced computing resources located at iVEC@ARRC and on parallel clusters acquired through the WCU program in the School of Mechanical and Aerospace Engineering at SNU.

\section{References}

[1] Mélinon P, Masenelli B, Tournus F and Perez A 2007 Playing with carbon and silicon at the nanoscale Nature Mater. 6 479-90

[2] Han X D, Zhang Y F, Liu X Q, Zhang Z, Hao Y J and Guo X Y 2005 Lattice bending, disordering, and amorphization induced plastic deformation in a SiC nanowire J. Appl. Phys. 98124307

[3] Han X D, Zhang Y F, Zheng K, Zhang X N, Zhang Z, Hao Y J, Guo X Y, Yuan J and Wang Z L 2007 Low-temperature in situ large strain plasticity of ceramic $\mathrm{SiC}$ nanowires and its atomic-scale mechanism Nano Lett. 7 452-7

[4] Zhang Y F, Han X D, Zheng K, Zhang Z, Zhang X N, Fu J Y, Ji Y, Hao Y J, Guo X Y and Wang Z L 2007 Direct observation of super-plasticity of beta-SiC nanowires at low temperature Adv. Funct. Mater. 17 3435-40

[5] Kikuchi H, Kalia R K, Nakano A, Vashishta P, Branicio P S and Shimojo F 2005 Brittle dynamic fracture of crystalline cubic silicon carbide (3C-SiC) via molecular dynamics simulation J. Appl. Phys. 98103524

[6] Makeev M A, Srivastava D and Menon M 2006 Silicon carbide nanowires under external loads: an atomistic simulation study Phys. Rev. B 74165303

[7] Shin C J, Meyers M A, Nesterenko V F and Chen S J 2000 Damage evolution in dynamic deformation of silicon carbide Acta Mater. 48 2399-420

[8] Zhang T Y, Luo M and Chan W K 2008 Size-dependent surface stress, surface stiffness, and Young's modulus of hexagonal prism [111] $\beta$-SiC nanowires J. Appl. Phys. 103104308 
[9] Wang Z G, Zu X T, Li Z J and Gao F 2008 Amorphous layer coating induced brittle to ductile transition in single crystalline SiC nanowires: an atomistic simulation J. Phys. D: Appl. Phys. 41155419

[10] Perisanu S, Gouttenoire V, Vincent P, Ayari A, Choueib M, Bechelany M, Cornu D and Purcell S T 2008 Mechanical properties of $\mathrm{SiC}$ nanowires determined by scanning electron and field emission microscopies Phys. Rev. B 77165434

[11] Wang Z L, Dai Z R, Gao R P, Bai Z G and Gole J L 2000 Side-by-side silicon carbide-silica biaxial nanowires: Synthesis, structure, and mechanical properties Appl. Phys. Lett. 77 3349-51

[12] Wang Z L, Dai Z R, Gao R P and Gole J L 2002 Measuring the Young's modulus of solid nanowires by in situ TEM J. Electron Microsc. 51 S79-85

[13] Wong E W, Sheehan P E and Lieber C M 1997 Nanobeam mechanics: elasticity, strength, and toughness of nanorods and nanotubes Science 277 1971-5

[14] Ye H, Titchenal N, Gogotsi Y and Ko F 2005 SiC nanowires synthesized from electrospun nanofiber templates $A d v$. Mater. 17 1531-5

[15] Shim H W and Huang H C 2007 Three-stage transition during silicon carbide nanowire growth Appl. Phys. Lett. 90083106

[16] Kim Y W, Mitomo M and Nishimura T 2001 Heat-resistant silicon carbide with aluminium nitride and erbium oxide J. Am. Ceram. Soc. 84 2060-4

[17] Jin G Q, Liang P and Guo X Y 2003 Novel method for synthesis of silicon carbide nanowires J. Mater. Sci. Lett. 22 767-70

[18] Yang W, Araki H, Tang C, Thaveethavorn S, Kohyama A, Suzuki H and Noda T 2005 Single-crystal SiC nanowires with a thin carbon coating for stronger and tougher ceramic composites Adv. Mater. 17 1519-23

[19] Wu R B, Li B S, Gao M X, Chen J J, Zhu Q M and Pan Y 2008 Tuning the morphologies of SiC nanowires via the control of growth temperature, and their photoluminescence properties Nanotechnology 19335602

[20] Zhang Y F, Shim H W and Huang H C 2008 Size dependence of twin formation energy in cubic $\mathrm{SiC}$ at the nanoscale Appl. Phys. Lett. 92261908

[21] Baierle R J, Piquini P, Neves L P and Miwa R H 2006 Ab initio study of native defects in $\mathrm{SiC}$ nanotubes Phys. Rev. B 74155425

[22] Zhang B, Li J B, Sun J J, Zhang S X, Zhai H Z and Du Z W 2002 Nanometre silicon carbide powder synthesis and its dielectric behaviour in the GHz range J. Eur. Ceram. Soc. 22 93-9
[23] Wang J, Lu C, Wang Q, Xiao P, Ke F J, Bai Y L, Shen Y G, Liao X Z and Gao H J 2011 Understanding large plastic deformation of $\mathrm{SiC}$ nanowires at room temperature Europhys. Lett. 9563003

[24] Lindefelt U, Iwata H, Öberg S and Briddon P R 2003 Stacking faults in $3 \mathrm{C}-, 4 \mathrm{H}$, and $6 \mathrm{H}-\mathrm{SiC}$ polytypes investigated by an ab initio supercell method Phys. Rev. B 67155204

[25] Vashishta P, Kalia R K, Nakano A and Rino J P 2007 Interaction potential for silicon carbide: a molecular dynamics study of elastic constants and vibrational density of states for crystalline and amorphous silicon carbide J. Appl. Phys. 101103515

[26] Melchionna S, Ciccotti G and Holian B L 1993 Hoover NPT dynamics varying in shape and size Mol. Phys. 78 533-44

[27] Smith W, Yong C W and Rodger P M 2002 DL_POLY: application to molecular simulation Mol. Simul. 28 385-471

[28] Zhou M 2003 A new look at the atomic level virial stress: on continuum-molecular system equivalence Proc. R. Soc. A 459 2347-92

[29] Wang J, Kulkarni A J, Ke F J, Bai Y L and Zhou M 2008 Novel mechanical behaviour of $\mathrm{ZnO}$ nanorods Comput. Methods Appl. Mech. Eng. 197 3182-9

[30] Chan W K, Luo M and Zhang T Y 2008 Molecular dynamics simulations of four-point bending tests on $\mathrm{SiC}$ nanowires Scr. Mater. 59 692-5

[31] Szlufarska I, Nakano A and Vashishta P 2005 A crossover in the mechanical response of nanocrystalline ceramics Science 309 911-4

[32] Ovid'ko I A 2011 Nanoscale multiplane shear and twin deformation in nanowires and nanocrystalline solids Appl. Phys. Lett. 99061907

[33] Lagos M J, Sato F, Galvão D S and Ugarte D 2011 Mechanical deformation of nanoscale metal rods: when size and shape matter Phys. Rev. Lett. 106055501

[34] Gao Y, Wang H, Zhao J, Sun C and Wang F 2011 Anisotropic and temperature effects on mechanical properties of copper nanowires under tensile loading Comput. Mater. Sci. $503032-7$

[35] Ji B H and Gao H J 2004 Mechanical properties of nanostructure of biological materials J. Mech. Phys. Solids 52 1963-90

[36] Liu Y, Wang B, Yoshino M, Roy S, Lu H and Komanduri R 2005 Combined numerical simulation and nanoindentation for determining mechanical properties of single crystal copper at mesoscale J. Mech. Phys. Solids 53 2718-41

[37] Wang J, Xiao P, Zhou M, Wang Z R and Ke F J 2010 Wurtzite-to-tetragonal structure phase transformation and size effect in ZnO nanorods J. Appl. Phys. 107023512 